\title{
Electric field-induced Nonlinear I-V Characteristic in a AgNWs/PVA Film Composite
}

\author{
Shi-Yang ZHAO, Qing-Guo WANG*, Xiao-Liang WANG, Pin LU, Zhao-Ming \\ QU, Wei CHENG, Li-Yun BAI and Yan WANG
}

Institute of Electrostatic \& Electromagnetic Protection, Ordnance Engineering College, Shijiazhuang, China

e-mail: 18243085974@163.com, qingguowang99@aliyun.com,wxIsr@126.com, 07041615@163.com, iamqzm3990@163.com,68649577@qq.com, 490322102@qq.com, katered198311@163.com

Keywords: silver nanowires; poly(vinyl alcohol); nonlinear current-voltage characteristic

\begin{abstract}
Uniform silver nanowires (AgNWs) were synthesized by a simple chemical reduction method with a typical diameter of $\sim 200 \mathrm{~nm}$ and a typical length of $\sim 6 \mathrm{um}$, and poly(vinyl alcohol)(PVA) aqueous solution was used to prepare AgNWs/PVA film. This combination shows good nonlinear current-voltage $(I-V)$ characteristic under relatively low applied voltage, the resistance of the film decreased strongly with increasing of the applied voltage, and the minimum value of resistance was $520 \Omega$ in the whole measurement. The quantum tunneling effect may be the main cause of the nonlinear $I-V$ characteristic of the AgNWs/PVA.
\end{abstract}

\section{Introduction}

Nano-scale metals have extraordinary mechanical, magnetic, electrical and optical properties comparing to bulk metal because of their quantum size effect, quantum tunneling effect, and large specific surface area. Metal nanowires have received great interest and attention in recent years, duo to their potential use as optical, electronic, flexible and stretchable devices[1]. Silver nanowires(AgNWs) have highest electrical and thermal conductivities among all metals and wonderful catalytic, optical and photonic properties[2]. Many researchers have reported the preparation approaches of AgNWs, including polyol method[3-5], microwave-polyol method[6], electrochemical method[7], solvothermal method[8], ultraviolet irradiation[9], wet chemical synthesis[10], seed catalysis process[11], oil-water-air interface self-assembly method[12], template-directed synthesis method[13]. Some progress have been acquired in the study of electrically conductive properties of polymer matrix filling with metal filler, the percolation threshold of wire-like metal filler is lower than nanoparticle filler in the condition of obtaining the same conductivity[14], and wire-like filler with high aspect ratio filling polymer can be decreased than low aspect ratio filler[15]. In this way, the amount of filler can be reduced. Field-induced nonlinear electrically conductive characteristic was observed when the filler volume fraction is close to percolation threshold. Researchers have deserved to study the nonlinear conductive mechanism of composites for decades and proposed that quantum tunneling effect is the cause. Polley et al. proposed that electron "jump" across the gap, with an exponential dependence on gap[16]. Simmons proposed a generalized formula for the electric tunnel effect between similar electrodes separated by a thin insulating film[17]. Ping Sheng et al. suggested that nonlinear conductive mechanism is tunneling with potential-barrier modulation by thermal fluctuations[18]. Beek and Van Pul proposed that electron passage in these systems is due to tunneling, regarded as a special case of internal field emission[19]. Sadie I. White et al. suggested that resistive switching in silver nanowires composites may be the result of the field-induced formation of conducting silver filaments[20]. Nonlinear conductive polymer composites have broad application prospects in electromagnetic protection, integrated circuits, nonvolatile memory, and other fields[21,22].

In this paper, AgNWs were prepared with a typical diameter of $\sim 200 \mathrm{~nm}$ and a typical length of $\sim 6$ um by simple chemical reduction method. As a important chemical material, poly(vinyl 
alcohol)(PVA) is safe and nontoxic, cheap and easy to obtain, has great forming performance and excellent mechanical properties. AgNWs were dispersed in PVA aqueous solution to prepare the homogeneous films, and the composite have great nonlinear conductive characteristic.

\section{Experimental}

\section{Materials}

Ethylene glycol(EG, analytical reagent(AR)) was purchased from Tianjin Yongda Chemical Reagent Company Limited, $\mathrm{FeCl}_{3}$ and poly(vinyl pyrrolidone)(PVP)(K88-96, Mw 1,300,000) were provided by Aladdin Industrial Corporation, silver nitrate $(\mathrm{AgNO} 3$ (purity $\geq 99.9 \%$ )) was purchased from Shanghai Fine Chemical Industry Material Research Institute, and PVA(purity $\geq 99.9 \%$ ) from Sinopharm Chemical Reagent Co. , Ltd. All chemicals and reagents are used without further purification. Deionized water was used throughout entire experiment.

\section{Preparation of AgNWs}

0.8g PVP was dissolved in 100ml EG and $1 \mathrm{~g}$ AgNO3 was added into PVP/EG solution to obtain solution A. $14 \mathrm{ml} \mathrm{FeCl} / \mathrm{EG}(600 \mathrm{mM})$ was poured into solution $\mathrm{A}$ under continuously vigorous magnetic stirring. Then the solution was added to a $250 \mathrm{ml}$ round bottom flask and heated at $130^{\circ} \mathrm{C}$ for 5 hours in a silicon bath. The faint yellow solution changed to brownish red and then turned to milk white. The mixture was allowed to be cooled to room temperature in air for 1 hour. To acquire purified AgNWs, the mixture was diluted with acetone, deionized water and ethanol, washed and centrifuged 3 times to remove excess of EG and other reaction product. In the whole reaction, the EG acts as both reducing agent and solvent, surfactants such as PVP could control the growth rates of various faces of silver by coordinating to the surfaces, lead silver to grow into uniform nanowires, $\mathrm{FeCl}_{3}$ as mediated and nucleation agent can react with and remove the adsorbed atomic oxygen from silver surfaces[23,24]. Fig. 1 shows scanning electron microscopy (SEM, S-4800) image of AgNWs.

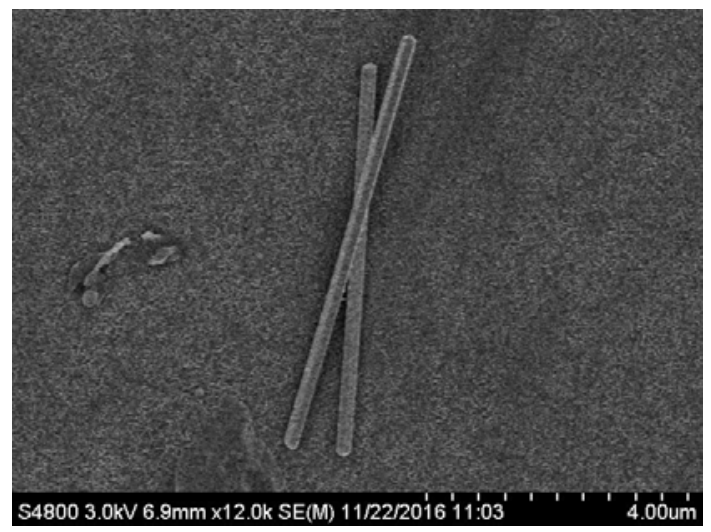

Figure 1. SEM images for AgNWs with a typical diameter of $\sim 200 \mathrm{~nm}$ and a typical length of $\sim 6$ um.

\section{Preparation of AgNWs/PVA}

$5 \mathrm{~g}$ PVA was dissolved in $50 \mathrm{ml}$ deionized water at $90^{\circ} \mathrm{C}$ in a round bottom flask under continuously vigorous magnetic stirring. An appropriate amount of AgNWs was added to the flask and stirred for 24h. The prepared composite solution was casted to Petri-dishes, solid composite was peeled off after the evaporation of the solvent at room temperature in air. The sample was processed into a $4 \mathrm{~cm} \times 2 \mathrm{~cm} \times 300 \mathrm{~nm}$ film, the effective test section of the film has dimensions of $\sim 2 \mathrm{~cm} \times 2 \mathrm{~cm} \times 300 \mathrm{~nm}$. Fig. 2 shows SEM(KYKY-EM6200) image of the solid composite, represents the high degree of nanowires dispersion. Inductively Coupled Plasma Source Mass Spectrometer(ICP) was used to determine the content of AgNWs. The measured concentration of AgNWs in composite was showed in Table 1. 


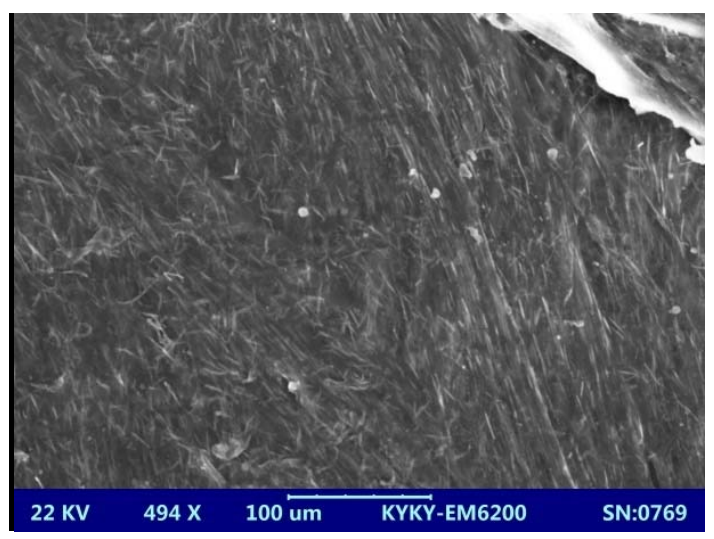

Figure 2. The good dispersion observed in AgNWs/PVA composite.

Table 1 The Concentration Of AgNWs In Composite

\begin{tabular}{c|c}
\hline Sample & Concentration of AgNWs in composite \\
\hline AgNWs/PVA composite & $5.58 \mathrm{wt} \%$ \\
\hline
\end{tabular}

\section{Results and Discussion}

For resistance measurement on the film at room temperature, the circuit shown in Fig. 3 is used. The $\delta$ (conductivity) values of the film were calculated by:

$$
\delta=\frac{l}{R S}
$$

In which $l, S, R$ is the length, area of the cross section and resistance, respectively. As for resistance $R$, is described by

$$
R=\frac{U R_{1}-U_{1} R_{2}-U_{1} R_{1}}{U_{1}}
$$

Where $U$ is the value of DC applied voltage, $U_{1}$ is the voltage across the resistor, the values of $R_{2}$ and $R_{1}$ are $3 \mathrm{M} \Omega, 1 \mathrm{~K} \Omega$, respectively.

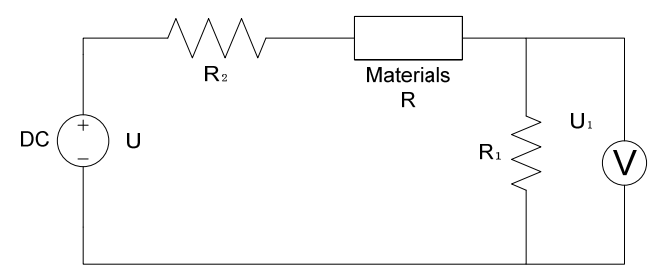

Figure 3. Circuit for resistance measurement.

As we can see through the characteristic curve(Fig. 4), electrically conductive properties of AgNWs/PVA film gradually increase with the increasing of the applied voltage. Table 2 is the measurement data analysis of AgNWs/PVA film. Conductivity of Sample changes from insulator to conductor in the range of $0.6 \sim 0.7 \mathrm{MV} / \mathrm{m}$, the resistance changes by a factor of $2 \times 10^{3}$, and conductivity increases by 560 times. 


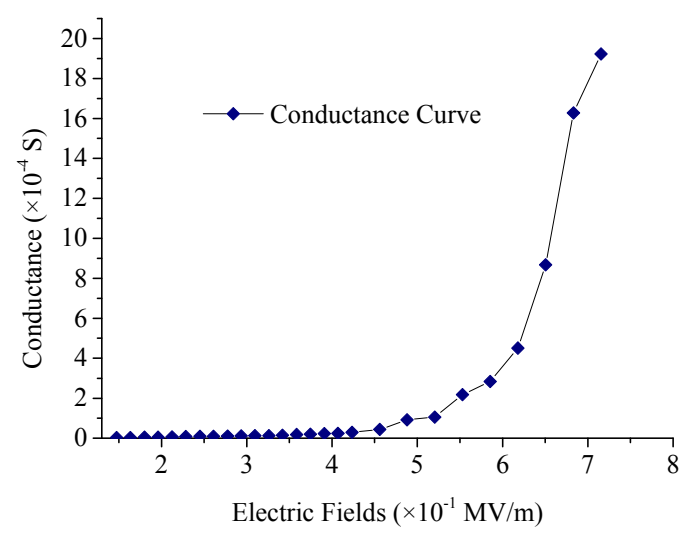

Figure 4. Conductance of AgNWs/PVA film.

Table 2 Measurement Data Analysis of AgNWs/PVA Film

\begin{tabular}{c|c|c}
\hline Voltage $(\mathrm{kV})$ & $\begin{array}{c}\text { Average electric field } \\
(\mathrm{MV} / \mathrm{m})\end{array}$ & Electrically conductive properties \\
\hline $0.09 \sim 2.63$ & $\leq 0.1315$ & No current \\
$2.59 \sim 8.47$ & $0.1475 \sim 0.4235$ & Started conduction, weak current \\
$9.12 \sim 12.36$ & $0.456 \sim 0.618$ & The conductivity increased rapidly \\
$13.01 \sim 14.31$ & $0.6505 \sim 0.7155$ & The conductance is maximized and the \\
resistance was reduced to $520 \Omega$
\end{tabular}

The relationship between electrical conductance and electric field was obtained by fitting the measurement data:

$$
G=A+\frac{A-B}{1+10^{\left(\log ^{M}-x\right) \times N}}
$$

Where $A=0.00284, B=1.73973 * 10^{-5}, M=4.79193, N=10.30861$.

When the applied electric field was $0.2856 \mathrm{MV} / \mathrm{m}$, that is the voltage was about $6 \mathrm{kV}$, The film began to have a weak current; The current through the film significantly increased with the applied voltage of $11 \mathrm{kV}$; conductance of the film was closed to the conductor when the applied voltage reached to $13 \mathrm{kV}$, and the current further increased stably.

The nonlinear $I-V$ characteristic was first observed in AgNWs/PVA composite. The composite showed ohmic behavior when the applied voltage was low, and nonlinear $I-V$ characteristic was showed with gradually increasing voltage. Duo to the conductive filler separated by insulting matrix, it is impossible for the film to form electrical network under relatively low voltage . When the applied voltage was gradually increasing to an appropriate value, a number of electrons were activated to form conductive path, the conductivity of the material was improved instantly. As for possible conductive mechanism, we proposed that a stream of electrons hopping through the potential barrier, and tunneling takes place only when neighboring AgNWs were very close.

\section{Conclusion}

In conclusion, chemical method was used to synthesize the uniform AgNWs, AgNWs/PVA film having good dispersion was prepared by simple mixing and magnetic stirring, and the $I-V$ behavior of the film was investigated. The film exhibited wonderful nonlinear $I-V$ characteristic, and conductive process was described. We believe that local electric field must be sufficiently large to drive the electron to hop through the gap between the neighboring AgNWs. We will systematically explore the effects of different aspect ratios and loading content of AgNWs on the nonlinear $I-V$ 
characteristics of the composites, and study the effects of different polymer matrix on conductivity of composites.

\section{References}

[1] Jinting Jiu, Katsuaki Suganuma. Metallic nanowires and their application[J]. IEEE Transactions on Components, Packaging and Manufacturing Technology, 2016, 1-19.

[2] Xiong-Zhi Xiang, Wen-Ya Gong, Ming-Sheng Kuang, et al. Progress in application and preparation of silver nanowires[J]. Rare Metals,2016, 35(4): 289-298.

[3] Sun Y, Gates B, Mayers B, et al. Crystalline silver nanowires by soft solution processing[J]. Nano letters, 2002, 2(2): 165-168.

[4] Zhang W, Chen P, Gao Q, et al. High-concentration preparation of silver nanowires: restraining in situ nitric acidic etching by steel-assisted polyol method[J]. Chemistry of Materials, 2008, 20(5): 1699-1704.

[5] Gao Y, Jiang P, Liu D F, et al. Evidence for the monolayer assembly of poly (vinylpyrrolidone) on the surfaces of silver nanowires[J]. The Journal of Physical Chemistry B, 2004, 108(34): 12877-12881.

[6] Tsuji M, Matsumoto K, Miyamae N, et al. Rapid preparation of silver nanorods and nanowires by a microwave-polyol method in the presence of Pt catalyst and polyvinylpyrrolidone[J]. Crystal growth \& design, 2007, 7(2): 311-320.

[7] Zheng X J, Jiang Z Y, Xie Z X, et al. Growth of silver nanowires by an unconventional electrodeposition without template[J]. Electrochemistry communications, 2007, 9(4): 629-632.

[8] Chen D, Qiao X, Qiu X, et al. Large-scale synthesis of silver nanowires via a solvothermal method[J]. Journal of Materials Science: Materials in Electronics, 2011, 22(1): 6-13.

[9] Zou K, Zhang X H, Duan X F, et al. Seed-mediated synthesis of silver nanostructures and polymer/silver nanocables by UV irradiation[J]. Journal of Crystal Growth, 2004, 273(1): 285-291.

[10]Caswell K K, Bender C M, Murphy C J. Seedless, surfactantless wet chemical synthesis of silver nanowires[J]. Nano Letters, 2003, 3(5): 667-669.

[11]Chen C, Wang L, Yu H, et al. Morphology-controlled synthesis of silver nanostructures via a seed catalysis process[J]. Nanotechnology, 2007, 18(11): 115612.

[12] Shi H Y, Hu B, Yu X C, et al. Ordering of disordered nanowires: spontaneous formation of highly aligned, ultralong Ag nanowire films at oil-water-air interface[J]. Advanced Functional Materials, 2010, 20(6): 958-964.

[13] Storhoff J J, Mirkin C A. Programmed materials synthesis with DNA[J]. Chemical Reviews, 1999, 99(7): 1849-1862.

[14]Carmona F, Barreau F, Delhaes P, et al. An experimental model for studying the effect of anisotropy on percolative conduction[J]. Journal de Physique Lettres, 1980, 41(22): 531-533.

[15] White S I, Mutiso R M, Vora $\mathrm{P} \mathrm{M}$, et al. Electrical percolation behavior in silver nanowire-polystyrene composites: simulation and experiment[J]. Advanced Functional Materials, 2010, 20(16): 2709-2716.

[16]Polley M H, Boonstra B. Carbon blacks for highly conductive rubber[J]. Rubber Chemistry and Technology, 1957, 30(1): 170-179.

[17] Simmons. J G. Generalized formula for the electric tunnel effect between similar electrodes 
separated by a thin insulating film[J]. J. Appl. Phys, 1963, 34(6): 1.

[18] Sheng P, Sichel E K, Gittleman J I. Fluctuation-induced tunneling conduction in carbon-polyvinylchloride composites[J]. Physical Review Letters, 1978, 40(18): 1197.

[19]Van Beek L K H, Van Pul B. Internal field emission in carbon black - loaded natural rubber vulcanizates[J]. Journal of Applied Polymer Science, 1962, 6(24): 651-655.

[20] White S I, Vora P M, Kikkawa J M, et al. Resistive switching in bulk silver nanowire-polystyrene composites[J]. Advanced Functional Materials, 2011, 21(2): 233-240.

[21]Pande S, Chaudhary A, Patel D, et al. Mechanical and electrical properties of multiwall carbon nanotube/polycarbonate composites for electrostatic discharge and electromagnetic interference shielding applications[J]. Rsc Advances, 2014, 4(27): 13839-13849.

[22]Feng P A N, Chao C, Wang Z, et al. Nonvolatile resistive switching memories-characteristics, mechanisms and challenges[J]. Progress in Natural Science: Materials International, 2010, 20: $1-15$.

[23] Sun Y, Xia Y. Large-scale synthesis of uniform silver nanowires through a soft, self-seeding, polyol process[J]. Nature, 1991, 353(1991): 737.

[24]Satoungar M T, Azizi H, Fattahi S, et al. Effect of Different Mediated Agents on Morphology and Crystallinity of Synthesized Silver Nanowires Prepared by Polyol Process[J]. Journal of Nanomaterials, 2016, 2016: 5. 